\section{Die Zukunft, die nie eintrat}

\author{
Über die Nützlichkeit filmischer \\ Zukunftsbilder des autonomen Fahrens \\ für die Prospektive
}

\begin{abstract}
von Fabian Kröger, Université Paris 1 Panthéon Sorbonne sowie HumboldtUniversität zu Berlin
\end{abstract}

Das fahrerlose Automobil gehört zu den Verkehrszukünften, die seit fast einem Jahrhundert der Realisierung harren. Das automatische Fahren begleitet uns als Traum und ist deshalb stark bildbasiert. Es lässt sich zeigen, dass in vielen Illustrationen dieser Verkehrsvision das Soziale statisch bleibt, besonders die Geschlechterverhältnisse werden anachronistisch dargestellt. Science Fiction-Filmen gelingt es besser, den möglichen gesellschaftlichen Kontext autonomen Fahrens zu imaginieren. Dies macht sie für die Prospektive interessant. So eignet sich das Kino für eine Futurologie zukünftiger Mentalitäten und Emotionen. Indem es mögliche zukünftige Lüste und Ängste vorstellbar macht, beeinflusst es auch Akzeptanzbildung und Produktgestaltung.

Historisch bedeutsame Veränderungen im Bereich der Mobilität wurden von den jeweiligen Zeitgenossen oft unterschätzt: Das Automobil sei eine Modeerscheinung, die Zukunft gehöre dem Pferd, sagte Kaiser Wilhelm II. im Jahre 1904. Sogar Gottlieb Daimler vermutete, das Auto werde am Mangel an Chauffeuren scheitern. Und wegen seiner hohen Geschwindigkeit wurde das Eisenbahnfahren im 19. Jahrhundert verdächtigt, der Gesundheit zu schaden.

Während diese Anekdoten häufig kolportiert werden, sind die überschätzten, gescheiterten, verworfenen oder verzögerten Verkehrsvisionen weniger im öffentlichen Bewusstsein präsent. Die Zeit des wirtschaftlichen Aufschwungs nach dem 2. Weltkrieg war die Hochphase dieser Techno-Träume, vor allem in den USA. So galten Autos mit Atomantrieb (Ford Nucleon 1958) als machbar. Beat-Poet William Burroughs erinnerte in den frühen 1970er Jahren per Graffiti an New Yorker Hauswänden an eine weitere nie realisierte Mobilitätsvision: „Wo sind die
Individual-Hubschrauber geblieben, die man uns versprochen hat?" (Agentur Bilwet 1993, S. 8f.).

\section{Die Bildgeschichte des Traums vom autonomen Fahren}

Auch der Traum eines selbstfahrenden, fahrerlosen Automobils gehört in diese Reihe, unterscheidet sich von den gerade genannten Entwürfen aber in zwei Punkten:

Überraschend ist erstens, dass die Vision des autonomen Fahrens schon seit 1925 existiert, ohne dass sie in größerem Stil realisiert worden wäre. Mehr noch: Das fahrerlose Automobil liegt seit 80 Jahren immer 20 Jahre in der Zukunft. Seit Langem erwarten wir also eine kurzfristige Realisierung dieser Idee. Diese merkwürdige Dynamik permanenter Verschiebung ist nicht damit zu erklären, dass niemand das automatische Fahren braucht. ${ }^{1}$ Der Historiker Joseph J. Corn erinnert vielmehr daran, dass die Geschichte der Zukunft eine Geschichte menschlicher Hoffnungen und Ängste ist (Corn 1986b, S. 219). Das bedeutet, dass Zukunftsvisionen wie das autonome Fahren in erster Linie als Traum gebraucht werden. Darin liegt ihr eigentlicher kultureller, vor allem aber auch ökonomischer Wert. Nicht nur während der Großen Depression der 1930er Jahre wirkten sich Zukunftsversprechen in Werbung und Produktdesign günstig auf die Verkaufszahlen aus (Corn 1986a, S. 4).

Zweitens lässt das sonderbare Zeitverhältnis der Dauerutopie des autonomen Fahrens Bildern eine besondere Rolle zukommen. Das kontinuierlich nicht-präsente, bessere Morgen muss zumindest als Bild präsent gemacht werden, um vorstellbar zu werden und als historisch dauerhaftes Leitbild fungieren zu können. So überrascht es nicht, dass das erste tatsächlich selbstfahrende Automobil 1935 als Leinwandgefährt eines USVerkehrserziehungsfilms (The Safest Place) die Bühne betrat. ${ }^{2}$ Im Zuge der populärkulturellen Vermittlung dieser Vision entstanden zahlreiche Illustrationen in Magazinen wie Popular Science, Popular Mechanix etc., deren Bildsprache und Funktion bereits an anderer Stelle untersucht wurde (Kröger 2012). Auffällig ist in der gesamten Bildgeschichte des autonomen Fahrens das ungleiche Verhältnis von Technik und Sozialem. 


\section{Das Soziale der Zukunftsbilder bleibt meist statisch}

Eine Anzeige aus einer Ausgabe des LIFE-Magazines von 1956 zeigt im Vordergrund eine große Limousine, die auf der Mittelspur eines hellen Autobahnbandes dahinrollt, das sich bis zum Horizont erstreckt (s. Abb. 1). Die zentralperspektivische Bildkonstruktion ist ein, seit den 1930er Jahren immer wiederkehrender, visueller Archetyp oder Visiotyp (Pörksen 1997) der Inszenierung des fahrerlosen Automobils. Einem kulturellen Muster folgen aber auch die sozialen Elemente der Anzeige: Eine vierköpfige Familie sitzt um einen Tisch herum, streng eingeschmiedet in die gesellschaftlichen Konventionen, die hegemonialen Werte der 1950er Jahre. Der Vater besetzt den Fahrersitz, auch wenn er sich vom Lenkrad abgewandt hat. Mutter und Tochter spielen Domino, während der Sohn sein Modellflugzeug betrachtet.

Abb. 1: Anzeige Americas Independent Electric Light and Power Companies

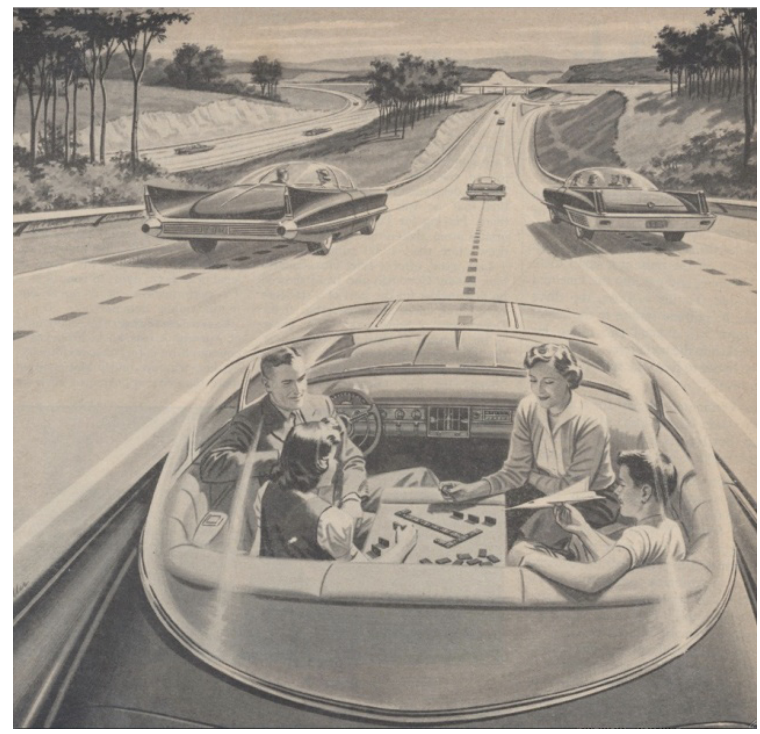

Quelle: LIFE Magazine 1956, S. 8; vgl. auch Kröger 2012, S. 104f.

Bis heute ist es die Regel, dass Zukunftsbilder die technischen Details neuer Gadgets sehr detailliert und phantasievoll darstellen, in der Imagination des gesellschaftlichen Kontextes aber statisch ihrer Gegenwart verhaftet bleiben (Benford 2012, S. 15). Sie lassen sich deshalb meist auf den ersten Blick datieren. Auch die literarische Sci- ence Fiction kombiniere oft „neue Technologien mit alten sozialen Mustern", bemerkt Karlheinz Steinmüller von der Foresight-Company (Steinmüller 2010, S. 29). Technik wird getrennt vom Sozialen konzipiert. Dass technische und soziale Dimensionen zusammen in ihrer Zukünftigkeit dargestellt werden, ist eher selten. Dies hat einen einfachen Grund. Kulturelle und soziale Innovationen sind deutlich schwieriger zu prognostizieren: Es ist sehr viel komplizierter, die Kultur der Zukunft zu beschreiben, als aus dem bisherigen Fortschritt der Chipentwicklung auf die Leistungsfähigkeit künftiger Computer zu schließen.

\section{Die prospektiven Qualitäten des Kinos}

Für die Prospektive ergibt sich aus diesem Manko umso mehr die Aufgabe, zukünftige Bedürfnisse, Wünsche und Träume zu imaginieren, also an einer Futurologie zukünftiger Mentalitäten zu arbeiten. Sie könnte bei dieser Aufgabe an die literarische und filmische Science Fiction anknüpfen, die - im Gegensatz zu vielen gezeichneten Illustrationen - das imaginative Potenzial besitzt, auch die emotionalen Selbstverständlichkeiten einer zukünftigen, technisch radikal veränderten Lebenswelt zu beschreiben und zu hinterfragen. Denn die simulierten Welten der Science Fiction haben die Kraft, uns zukünftigen Lüsten und Ängsten näher $\mathrm{zu}$ bringen, betont der Literaturwissenschaftler Raimar Zons (2004, S. 331). Dies könnte eine Form der „narrativen“ (Hoffmann/Mars 1992, S. 197) oder ästhetischen Technikfolgenabschätzung inspirieren, der es nicht darum geht, die Zukunft vorauszusehen. Sie erschafft eine imaginäre Welt, in der sie Tendenzen unserer Gegenwart weiterdenkt und mögliche Zukünfte skizziert. So hätte gute Science Fiction zwar nicht die Erfindung des Automobils, aber den Stau vorhersehen können, bemerkte Science Fiction-Autor Fred Pohl (vgl. Steinmüller 2010, S. 21). Offensichtlich kann Science Fiction die „Mach- und Umsetzbarkeit“" technischer Neuerungen sogar treffsicherer voraussehen als Technikexperten (vgl. Wise 1974, zit. in Steinmüller 2010, S. 19). Aus diesen Gründen liege es nahe, „SF auch für Foresight zu nutzen“, betont Steinmüller (2010, S. 22). Die Europäische Raumfahrtbehörde machte hier den Anfang und gab eine Studie in Auftrag, die Science Fiction- 
Filme systematisch auf Ideen untersuchte, die für die Raumfahrt nutzbar sind (ESA 2002).

Ihre eigentliche Qualität geht aber über die Frage hinaus, welche Entwicklungen sie richtig oder falsch vorhergesehen, welche sie über- oder unterschätzt hat. Science Fiction beeinflusst zukünftige Innovationen: Die Art und Weise, wie ein Science Fiction-Film automatische Fahrzeuge zeigt, beeinflusst über das kollektive Imaginäre direkt die Akzeptanzbildung und indirekt die Konstruktionsprozesse der Automobilhersteller. Da diese Filme Ideen über wünschenswerte oder zu vermeidende Zukünfte in Umlauf bringen, sind sie ,selbst ein Faktor der Zukunftsgestaltung“, so Steinmüller (2010, S. 22).

\section{Abb. 2: Autonomes Fahrzeug ohne Interface zum Selbststeuern}

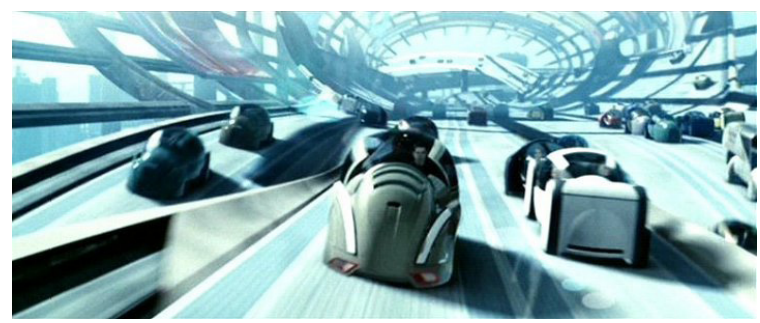

Quelle: Twentieth Century Fox, DreamWorks Pictures, Cruise/Wagner, 2002: Minority Report, USA. Regisseur: Steven Spielberg, Erstausstrahlung 2002; Screenshot aus: http:// inthemouthofdorkness.blogspot.de/2013/04/ matts-soapbox-city-of-future.html (download 24.4.14)

Als Beispiel sei der Film Minority Report (2002) von Steven Spielberg genannt, der automatische Fahrzeuge als Element einer Kontrollgesellschaft zeigt, in der Verbrechen verhindert werden können, bevor sie passieren. Ein Polizeioffizier, der beschuldigt wird, selbst in naher Zukunft einen Mord zu begehen, versucht in einem der selbstfahrenden Maglev-Vehikel zu flüchten (s. Abb. 2). Kurz darauf erhält das Fahrzeug aus der Verkehrszentrale den Befehl, umzukehren. Der Flüchtige muss aus dem Wagen springen, um zu entkommen. Diese Sequenz ist wichtig, da sie einen möglichen gesellschaftlichen Kontext autonomen Fahrens mit in das Bild der neuen Technologie hineinnimmt. Zugleich bleibt aber auch dieses Bild zeitgenössisch, da die modernen Gesellschaften aktuell mit den Grenzen staatlicher Überwachung hadern. Dennoch kann dieser Film der Foresight nützlich sein, da er an eine emotionale Qualität des Automobils erinnert, die historisch bedeutsam war: Seine Tauglichkeit als Fluchtfahrzeug.

\section{Ist das autonome Fahren seit den Google- Cars nicht mehr kinotauglich?}

Fragen wir nach der sozialen Einbettung künftiger Technologien, so müssen wir auch darauf achten, welche Objekte $a b$ einem bestimmten Moment nicht mehr im Kino gezeigt werden. Denn das Kino schwelgt gerne in Bildern, die in der außerfilmischen Realität keine massenhafte Entsprechung finden: Roland Barthes schrieb 1963, das amerikanische Kino habe aufgehört, „märchenhafte Interieurs" vorzuführen, als dem Durchschnittsamerikaner akzeptable Wohnverhältnisse zugänglich wurden (Barthes 2003, S. 61).

Heute lässt sich derselbe Mechanismus bei den selbstgesteuerten Fahrzeugen beobachten, die seit Ende der 1960er Jahre in verschiedenen Varianten das Kino bevölkerten: Seit dem Film I, Robot (2004) - also seit zehn Jahren - ist das automatische Automobil aus dem Spielfilm verschwunden. Warum? Eine klare Linie zwischen Ursache und Wirkung kann hier nicht gezogen werden, aber es ist doch bedeutsam, dass 2004, 2005 und 2007 drei vom US-Verteidigungsministerium finanzierte Roboterrennen stattfanden, in denen autonome Fahrzeuge gegeneinander antraten. Google rekrutierte die Mitarbeiter mehrerer Teams, die an dieser sog. DARPA-Challenge teilgenommen hatten und begann eine Flotte autonomer Fahrzeuge zu entwickeln, die auf öffentlichen Straßen hunderttausende Meilen ohne Eingriff des Fahrers - aber unter Dauerbeobachtung der Medien - zurückgelegt haben.

Damit ist die Vision des autonomen Fahrens vom „Märchenhaften in die Realität übergewechselt" (Barthes 2003, S. 61), ließe sich Roland Barthes paraphrasieren, der damit den Übergang des Autos vom Luxus- zum Alltagsobjekt beschrieb. Nachdem filmische und technische Visionen des fahrerlosen Automobils für einige Zeit gemeinsamen Vor-Bildern (Macho 2011) huldigten, scheinen sich ihre Wege nun wieder zu trennen. 
Dieses Fehlen filmischer Bilder kann als Symptom eines Ankommens des fahrerlosen Automobils in der realen Welt, vielleicht sogar gesellschaftlicher Akzeptanz analysiert werden. Offen bleibt allerdings die Frage, ob das fahrerlose Auto seinen Vor-Bildern aus den Spielfilmen ähneln wird oder ob es in viel banalerer Form in die Welt kommt - etwa als Seniorentransportmittel in Fußgängerzonen. Sicherlich werden die in der Realität angekommenen Testfahrzeuge den Konzeptionen der Science Fiction ganz andersartige Nach-Bilder zur Seite stellen, die wir heute noch nicht vorauszusehen vermögen.

\section{Anmerkungen}

1) Bis in die 1970er Jahre basierten alle Konzepte des automatischen Fahrens auf einer völligen Veränderung der Straßeninfrastruktur (Einbettung von Leitkabeln). Die damit verbundenen Kosten waren eines der Haupthindernisse auf dem Weg der Realisierung.

2) Schon 1925 fuhr ein fahrerloses Auto, ein 1926er Chandler mit dem Namen Linrrican Wonder über die Straßen von New York, entwickelt von der Firma Houdina Radio Control. Allerdings handelte es sich nicht um ein autonomes, sondern um ein ferngesteuertes Fahrzeug: Über eine Antenne erhielt es Signale eines Folgefahrzeugs, die dann über Elektromotoren in Fahrzeugmanöver übersetzt wurden.

\section{Literatur}

Agentur Bilwet, 1993: Medien-Archiv. Bensheim

Barthes, R., 2003: Mythologie des Automobils. In: NRW-Forum Kultur und Wirtschaft (Hg.): Autonom. Das Automobil in der zeitgenössischen Kunst. Ostfildern-Ruit, S. 59-69

Benford, G. (Hg.), 2012: The Wonderful Future that Never Was. New York

Corn, J.J., 1986a: Introduction. In: Corn, J.J. (Hg.): Imagining Tomorrow. History, Technology and the American Future. Cambridge, S. 1-9

Corn, J.J., 1986b: Epilogue. In: Corn, J.J. (Hg.): Imagining Tomorrow. History, Technology and the American Future. Cambridge, S. 219-229

ESA - European Space Agency (Hg.), 2002: Innovative Technologien aus der Science Fiction für weltraumtechnische Anwendungen. Noordwijk

Hoffmann, U.; Mars, L., 1992: Leitbildperspektiven. Technische Innovationen zwischen Vorstellung und Verwirklichung. In: Burmeister, K.; Steinmüller, K.,
(Hg.): Streifzüge ins Übermorgen. Zukunftsforschung und Science Fiction. Weinheim, S. 197-222

Kröger, F., 2012: Fahrerlos und unfallfrei. Eine frühe automobile Technikutopie und ihre populärkulturelle Bildgeschichte. In: Fraunholz, U.; Woschech, A. (Hg.): Technology Fiction - Technische Visionen und Utopien in der Hochmoderne. Bielefeld, S. 93-114

LIFE Magazine, 40/5, 30. Jg., 1956: Americas Independent Electric Light and Power Companies

Macho, T., 2011: Vorbilder. München

Pörksen, U., 1997: Weltmarkt der Bilder. Eine Philosophie der Visiotype. Stuttgart

Steinmüller, K., 2010: Science Fiction: Eine Quelle von Leitbildern für Innovationsprozesse und ein Impulsgeber für Foresight. In: Hauss, K.; Ulrich, S.; Hornbostel, S. (Hg.): Foresight - Between Science and Fiction. iFQ-Working Paper No. 7 (9/2010), S. 19-31

Twentieth Century Fox, DreamWorks Pictures, Cruise/ Wagner, 2002: Minority Report, USA. Regisseur: Steven Spielberg, Erstausstrahlung 2002; Screenshot aus: http://inthemouthofdorkness.blogspot.de/2013/04/ matts-soapbox-city-of-future.html (download 24.4.14) Wise, G., 1974: The Accuracy of Technological Forecasts, 1890-1940. In: Futures 10 (1974), S. 411-419

Zons, R., 2004: American Paranoia, Bladerunner/Matrix. In: Macho, T.; Wunschel, A. (Hg.): Science \& Fiction. Über Gedankenexperimente in Wissenschaft, Philosophie und Literatur. Frankfurt a. M.

\section{Kontakt}

M.A. Fabian Kröger

Equipe d'histoire des techniques, Institut d'histoire moderne et contemporaine (IHMC),

CNRS, Université Paris I Panthéon-Sorbonne

Centre Malher

9, rue Malher, 75004 Paris, Frankreich

E-Mail: fabian.kroger@gmail.com

$《 \gg$ 